This is an electronic reprint of the original article. This reprint may differ from the original in pagination and typographic detail.

Author(s): Repin, Sergey; Samrowski, Tatiana; Sauter, Stefan

Title: $\quad$ Estimates of the modeling error generated by homogenization of an elliptic boundary value problem

Year: $\quad 2016$

Version:

Please cite the original version:

Repin, S., Samrowski, T., \& Sauter, S. (2016). Estimates of the modeling error generated by homogenization of an elliptic boundary value problem. Journal of Numerical Mathematics, 24(1), 1-15. https://doi.org/10.1515/jnma-2014-1002

All material supplied via JYX is protected by copyright and other intellectual property rights, and duplication or sale of all or part of any of the repository collections is not permitted, except that material may be duplicated by you for your research use or educational purposes in electronic or print form. You must obtain permission for any other use. Electronic or print copies may not be offered, whether for sale or otherwise to anyone who is not an authorised user. 
Sergey Repin*, Tatiana Samrowski, and Stefan Sauter

\title{
Estimates of the modeling error generated by homogenization of an elliptic boundary value problem
}

\begin{abstract}
In this paper, we derive a posteriori bounds of the difference between the exact solution of an elliptic boundary value problem with periodic coefficients and an abridged model, which follows from the homogenization theory. The difference is measured in terms of the energy norm of the basic problem and also in the combined primal-dual norm. Using the technique of functional type a posteriori error estimates, we obtain two-sided bounds of the modeling error, which depends only on known data and the solution of the homogenized problem. It is proved that the majorant with properly chosen arguments possesses the same convergence rate, which was established for the true error. Numerical tests confirm the efficiency of the estimates.
\end{abstract}

Keywords: periodic structures, homogenization, elliptic boundary value problems, a posteriori error estimates, modeling error

MSC 2010: 35J15, 35B27, 65N15

DOI: $10.1515 /$ jnma-2014-1002

Received January 26, 2014; revised November 21, 2014; accepted January 28, 2015

\section{Introduction}

Boundary value problems with periodic structures arise in various applications. Homogenization theory is the major tool used to quantitatively analyze media with periodic structures. Within the framework of the theory (see, e.g., [9, 14]), the behavior of a heterogeneous media is described with the help of a certain homogenized problem, which is typically a boundary value problem with smooth coefficients, and the solution of a specially constructed problem with periodic boundary conditions. It has been proved that the functions reconstructed by this procedure converge to the exact solution as the cell size $\varepsilon$ tends to zero. Moreover, known a priori error estimates qualify the convergence rate in terms of $\varepsilon$. The goal of this paper is to derive two-sided estimates of the modeling error generated by homogenization. In other words, we wish to estimate the difference between the exact solution of the original problem and its approximation obtained by the corresponding homogenized model.

Let $\Omega \subseteq \mathbb{R}^{d}$ be a bounded domain with Lipschitz boundary $\partial \Omega$, such that $\Omega=\bigcup_{\mathbf{i}} \Pi_{\mathbf{i}}^{\varepsilon}$, where

$$
\Pi_{\mathbf{i}}^{\varepsilon}=\mathbf{x}_{\mathbf{i}}+\varepsilon \widehat{\Pi}=\left\{\mathbf{x} \in \mathbb{R}^{d} \mid \frac{\mathbf{x}-\mathbf{x}_{\mathbf{i}}}{\varepsilon} \in \widehat{\Pi}\right\}
$$

is the basic 'cell' (repeating element of the periodic structure, see Fig. 1), which is a simply connected domain with Lipschitz boundary, $\mathbf{x}_{\mathbf{i}}$ is the reference point of $\Pi_{\mathbf{i}}^{\varepsilon}$, and $\varepsilon$ is a small parameter (geometrical size of a cell). Here and later on, $\mathbf{x}$ denotes the global (Cartesian) coordinate system in $\mathbb{R}^{d}$ and $\mathbf{i}=\left(i_{1}, i_{2}, \ldots, i_{d}\right)$ denotes the counting multi-indices for the cells. The notations $\bigcup_{\mathbf{i}}$ and $\sum_{\mathbf{i}}$ are shorthands for the union and summation over all cells. It is assumed that the overall amount of $\Pi_{\mathbf{i}}^{\varepsilon}$ in $\Omega$ is bounded from above by the

\footnotetext{
*Corresponding Author: Sergey Repin: V. A. Steklov Institute of Mathematics, Fontanka 27, 191011 St. Petersburg, Russia, and University of Jyväskylä, Finland. Email: repin@pdmi.ras.ru

Tatiana Samrowski: Institute for Applied Mathematics and Physics, Zurich University of Applied Sciences, Technikumstrasse 6, $\mathrm{CH}-8400$ Winterthur, Switzerland

Stefan Sauter: Institut für Mathematik, Universität Zürich, Winterthurerstrasse 190, CH-8057 Zurich, Switzerland
} 

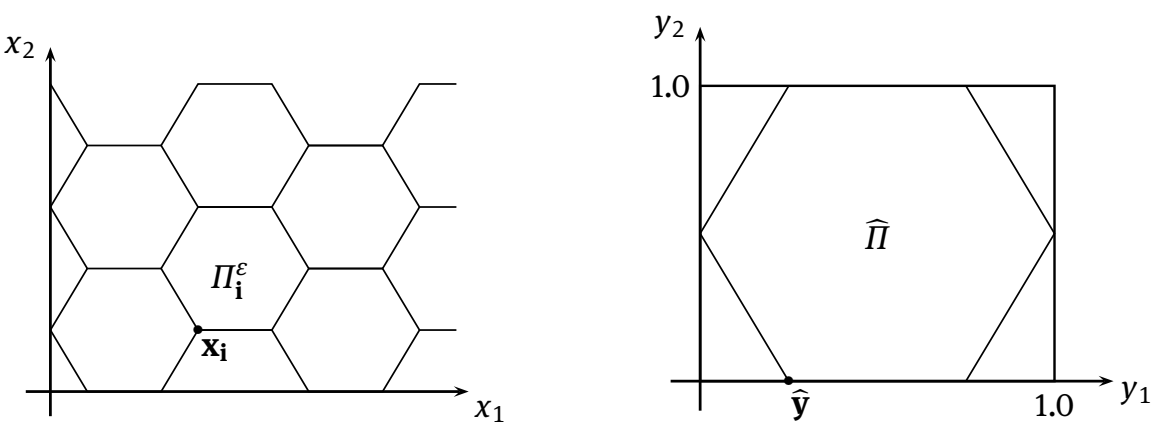

Figure 1. Periodic structure (left) and its basic cell (right).

quantity

$$
c_{0} \varepsilon^{-d}, \quad c_{0}=\mathcal{O}(1)
$$

and the diameter of $\Pi_{\mathbf{i}}^{\varepsilon}$ satisfies the relation

$$
\operatorname{diam} \Pi_{\mathbf{i}}^{\varepsilon}=\rho \varepsilon
$$

where $\rho$ is a parameter depending on the geometry of the cell. Usually, $\rho$ is easy to find (e.g., for a cubic cell $\rho=\sqrt{d})$.

In the basic cell (see Fig. 1), we use local Cartesian coordinates $\mathbf{y} \in \mathbb{R}^{d}$. For any $\Pi_{\mathbf{i}}^{\varepsilon}$, local and global coordinates are joined by the relation

$$
\mathbf{y}=\frac{\mathbf{x}-\mathbf{x}_{\mathbf{i}}}{\varepsilon} \in \widehat{\Pi} \quad \forall \mathbf{x} \in \Pi_{\mathbf{i}}^{\varepsilon}, \quad \forall \mathbf{i}
$$

On $\widehat{\Pi}$, we define a matrix function $\widehat{A} \in L^{\infty}\left(\widehat{\Pi}, \mathbb{R}_{\mathrm{sym}}^{d \times d}\right)$, where $\mathbb{R}_{\mathrm{sym}}^{d \times d}$ denotes the set of symmetric $d \times d$ matrices. We assume that

$$
c_{1}|\xi|^{2} \leqslant \widehat{A}(\mathbf{y}) \xi \cdot \xi \leqslant c_{2}|\xi|^{2} \quad \forall \xi \in \mathbb{R}^{d}, \quad \forall \mathbf{y} \in \widehat{\Pi}
$$

where $0<c_{1} \leqslant c_{2}<\infty$ and introduce the 'global' matrix

$$
A_{\varepsilon}(\mathbf{x}):=\widehat{A}\left(\frac{\mathbf{x}-\mathbf{x}_{\mathbf{i}}}{\varepsilon}\right) \quad \forall \mathbf{x} \in \Pi_{\mathbf{i}}^{\varepsilon}, \quad \forall \mathbf{i}
$$

which defines the periodic structure on $\Omega$. In view of (1.3), $A_{\varepsilon}$ (and its inverse counterpart $A_{\varepsilon}^{-1}$ ) satisfy similar two-sided estimates for any $\varepsilon$.

Consider the second-order elliptic equation

$$
-\operatorname{div}\left(A_{\varepsilon} \nabla u_{\varepsilon}\right)=f \quad \text { in } \Omega, \quad f \in L^{2}(\Omega)
$$

with homogeneous Dirichlet boundary conditions. The corresponding generalized solution $u_{\varepsilon} \in H_{0}^{1}(\Omega)$ is defined by the relation

$$
\int_{\Omega} A_{\varepsilon} \nabla u_{\varepsilon} \cdot \nabla w=\int_{\Omega} f w \quad \forall w \in H_{0}^{1}(\Omega) .
$$

For any $\varepsilon>0$, the solution $u_{\varepsilon}$ exists and is unique.

For a function $\zeta \in L^{1}(\omega)$, where $\omega$ is a measurable subset of $\Omega$, we define the mean value by

$$
\langle\zeta\rangle_{\omega}:=\frac{1}{|\omega|} \int_{\omega} \zeta
$$

If no confusion may arise, we omit in integrals the symbol of the corresponding Lebesque measure (e.g., $\mathrm{d} \mathbf{x})$. However, we write the measure explicitly if it is necessary to distinguish between integration over the global and local coordinates (as in Lemma 2.1). 
If we write $\int_{\omega}\langle\zeta\rangle_{\omega}$, then the average is considered as a constant function on $\omega$ (for vector-valued functions, we apply this definition componentwise). The error caused by the averaging (1.7) is denoted by

$$
\delta_{\omega} \zeta:=\left\|\zeta-\langle\zeta\rangle_{\omega}\right\|_{\omega}
$$

where $\|\cdot\|_{\omega}$ denotes the standard $L^{2}$-norm on $\omega$.

For a vector $\boldsymbol{\mu}=\left(\mu_{i}\right)_{i=1}^{d} \in\left(\mathbb{R}_{>0}\right)^{d}$ and $s \in \mathbb{R}, \boldsymbol{\mu}^{s}$ denotes the componentwise application of the power $s$, i.e., $\boldsymbol{\mu}^{s}=\left(\mu_{i}^{s}\right)_{i=1}^{d}$. For vector-valued functions $\boldsymbol{\zeta}=\left(\zeta_{k}\right)_{k=1}^{d} \in L^{1}\left(\omega, \mathbb{R}^{d}\right)$ and $\boldsymbol{\varphi}=\left(\varphi_{k}\right)_{k=1}^{d} \in L^{1}\left(\Omega\right.$, $\left.\mathbb{R}^{d}\right)$, we define the local and piecewise constant averages by means of the relations

$$
\delta_{\omega} \zeta:=\left(\left\|\zeta_{k}-\left\langle\zeta_{k}\right\rangle_{\omega}\right\|_{\omega}\right)_{k=1}^{d}, \quad \delta_{\Omega}^{\mathrm{pw}} \boldsymbol{\varphi}:=\varepsilon^{d / 2}\left(\sum_{\mathbf{i}}\left\|\varphi_{k}-\left\langle\varphi_{k}\right\rangle_{\Pi_{\mathrm{i}}^{\varepsilon}}\right\|_{\Pi_{\mathrm{i}}^{\varepsilon}}\right)_{k=1}^{d}
$$

and

$$
\left(\delta_{\omega} \boldsymbol{\zeta}\right)^{2}:=\left(\left\|\zeta_{k}-\left\langle\zeta_{k}\right\rangle_{\omega}\right\|_{\omega}^{2}\right)_{k=1}^{d}, \quad\left(\delta_{\Omega}^{\mathrm{pw}} \boldsymbol{\varphi}\right)^{2}:=\varepsilon^{d}\left(\left(\sum_{\mathbf{i}}\left\|\varphi_{k}-\left\langle\varphi_{k}\right\rangle_{\Pi_{\mathbf{i}}^{e}}\right\|_{\Pi_{\mathbf{i}}^{\varepsilon}}\right)^{2}\right)_{k=1}^{d} .
$$

Within the framework of homogenization theory, an approximation of $u_{\varepsilon}$ is constructed by the following procedure (see, e.g., $[7,9,14]$ ). First, we define (for $k=1,2, \ldots, d$ ) the solutions $N_{k}$ of 'cell problems'

$$
\begin{gathered}
\operatorname{div}\left(\widehat{A} \nabla N_{k}\right)=(\operatorname{div} \widehat{A})_{k} \text { in } \widehat{\Pi} \\
N_{k} \text { is periodic in } \widehat{\Pi} \\
\int_{\widehat{\Pi}} N_{k}=0 .
\end{gathered}
$$

With the help of them, the homogenized matrix

$$
A_{0}=\langle\widehat{A}(I-\nabla \boldsymbol{N})\rangle_{\widehat{\Pi}}
$$

is defined. The function $u_{0} \in H_{0}^{1}(\Omega)$ such that

$$
\int_{\Omega} A_{0} \nabla u_{0} \cdot \nabla w=\int_{\Omega} f w \quad \forall w \in H_{0}^{1}(\Omega)
$$

provides a "coarse" approximation of $u_{\varepsilon}$. It is known that (see, e.g., [7]),

$$
u_{\varepsilon} \rightarrow u_{0} \quad \text { in } L^{2}(\Omega), \quad u_{\varepsilon} \rightarrow u_{0} \quad \text { in } H_{0}^{1}(\Omega) \text { for } \varepsilon \rightarrow 0 .
$$

However, it is necessary to construct a sequence of more accurate approximations, which converges in a stronger sense. For this purpose, the homogenization theory suggests to use advanced approximations

$$
w_{\varepsilon}^{1}(\mathbf{x}):=u_{0}(\mathbf{x})-\varepsilon \psi^{\varepsilon}(\mathbf{x}) N_{k}\left(\frac{\mathbf{x}-\mathbf{x}_{\mathbf{i}}}{\varepsilon}\right) \frac{\partial u_{0}(\mathbf{x})}{\partial x_{k}} \quad \forall \mathbf{x} \in \Pi_{\mathbf{i}}^{\varepsilon}, \quad \forall \mathbf{i}
$$

where $\psi^{\varepsilon}:=\min \{1, \operatorname{dist}(\mathbf{x}, \partial \Omega) / \varepsilon\}$ is a cutoff function.

To prove optimal a priori convergence rates for the modeling error

$$
e_{\varepsilon}^{\bmod }:=u_{\varepsilon}-w_{\varepsilon}^{1}
$$

we need some extra assumptions (see [14], p.28), namely,

$$
u_{0} \in W^{2, \infty}(\bar{\Omega})
$$

and

$$
\frac{\partial N_{k}}{\partial y_{j}} \in L^{\infty}(\widehat{\Pi})
$$

Then, it can be proved (see, e.g., [7], Remark 5.13; [10]; [14], p. 28) that the modeling error satisfies the asymptotic estimates:

$$
\left\|u_{\varepsilon}-w_{\varepsilon}^{1}\right\|_{H^{1}(\Omega)} \leqslant \widetilde{c} \sqrt{\varepsilon}
$$


and

$$
\left\|A_{\varepsilon} \nabla u_{\varepsilon}-\mathbf{v}_{\mathbf{0}}-\varepsilon \mathbf{v}_{\mathbf{1}}\right\| \leqslant \widehat{c} \sqrt{\varepsilon}
$$

where

$$
\begin{aligned}
\mathbf{v}_{\mathbf{0}} & :=\left(I-\operatorname{curl}_{\mathbf{y}} \widetilde{N}\right) \boldsymbol{\mu} \\
\boldsymbol{\mu} & :=\left\langle A_{0}^{-1}\left(I-\operatorname{curl}_{\mathbf{y}} \widetilde{N}\right)\right\rangle_{\overparen{\Pi}}^{-1} \nabla u_{0} \\
\mathbf{v}_{\mathbf{1}} & :=-\operatorname{curl}_{\mathbf{x}}(\widetilde{N} \boldsymbol{\mu})
\end{aligned}
$$

the $d \times d$ matrix $\widetilde{N}$ with columns $\widetilde{N}_{k}$ is the solution of the auxiliary problem

$$
\begin{gathered}
\operatorname{curl} A_{0}^{-1}\left(\operatorname{curl} \widetilde{N}_{k}(\mathbf{y})\right)=\operatorname{curl}\left(A_{0}^{-1}\right)_{k} \text { in } \widehat{\Pi} \\
\operatorname{div} \widetilde{N}_{k}=0 \\
\widetilde{N}_{k} \text { is periodic in } \widehat{\Pi} \\
\int_{\widehat{\Pi}} \widetilde{N}_{k}=0
\end{gathered}
$$

and the columns of the matrix $\operatorname{curl}_{\mathrm{y}} \widetilde{N}$ are given by $\operatorname{curl}_{\mathrm{y}} \widetilde{N}_{k}, k=1,2, \ldots, d$.

Numerical methods for homogenized problems are actively studied. Such questions as adaptivity and error indication are among the most important questions arising in quantitative analysis of periodical structures. Here, we first of all mention residual type error indicators that develop the ideas suggested in [2,3] for finite element approximations. Since our approach is based on a different technique, we will sketch here only briefly some relevant literature on residual based estimation and refer for a detailed review, e.g., to [13]. A posteriori error estimates for the heterogeneous multiscale discretization (HMM) of elliptic problems in a periodic setting can be found in [12, 17]. In [1], an a posteriori estimate of residual type for general, possibly non-periodic, diffusion tensors with micro-scales is presented while a residual-type a posteriori error estimate for more general diffusion tensors has been developed in [13]. Also, we mention the papers $[4,5,29,30]$, which are closely related to the topic.

Our goal is to deduce estimates of $e_{\varepsilon}^{\text {mod }}$ of a different type, which provide guaranteed and fully computable bounds of the modeling error. The corresponding error majorant uses the solution of the homogenized problem and, in addition, involves free functions and a function $\eta$ defined on the cell of periodicity. This freedom can be utilized for improving the efficiency of the corresponding error bounds. Besides, the functions obtained in this way provide efficient reconstructions of the flux. In general, the estimates have the form

$$
\mathcal{M}_{\ominus}\left(w_{\varepsilon}^{1} ; \Theta\right) \leqslant\left\|\nabla\left(u_{\varepsilon}-w_{\varepsilon}^{1}\right)\right\|_{A_{\varepsilon}} \leqslant \mathcal{M}_{\oplus}\left(w_{\varepsilon}^{1} ; \boldsymbol{\eta}, \boldsymbol{\lambda}, s\right)
$$

where

$$
\|\boldsymbol{q}\|_{A_{\varepsilon}}:=\left(\int_{\Omega} A_{\varepsilon} \boldsymbol{q} \cdot \boldsymbol{q}\right)^{1 / 2} .
$$

The majorant $\mathcal{M}_{\oplus}$ and a minorant $\mathcal{M}_{\ominus}$ are derived in Sections 2 and 3, respectively. Numerical tests are exposed in Section 4. They confirm the efficiency of the estimates.

\section{Upper bound of the modeling error}

First, we prove a subsidiary result, which states an upper bound of the $L^{2}$-product of a globally defined function and a periodic function defined on the cell.

Lemma 2.1. For all $\mathbf{g} \in L^{2}(\Omega)^{d}, \boldsymbol{\eta} \in L^{2}(\widehat{\Pi})^{d}$, and all $\boldsymbol{\lambda}=\left(\lambda_{d}\right)_{k=1}^{d} \in\left(\mathbb{R}_{>0}\right)^{d}$ it holds

$$
\sum_{\mathbf{i}} \int_{\Pi_{\mathbf{i}}^{\varepsilon}} \mathbf{g}(\mathbf{x}) \cdot \boldsymbol{\eta}\left(\frac{\mathbf{x}-\mathbf{x}_{\mathbf{i}}}{\varepsilon}\right) \mathrm{d} \mathbf{x} \leqslant|\Omega|\langle\mathbf{g}\rangle_{\Omega} \cdot\langle\boldsymbol{\eta}\rangle_{\widehat{\Pi}}+\frac{\boldsymbol{\lambda}}{2} \cdot\left(\delta_{\Omega}^{\mathrm{pw}} \mathbf{g}\right)^{2}+\frac{\boldsymbol{\lambda}^{-1}}{2} \cdot\left(\delta_{\overparen{\Pi}} \boldsymbol{\eta}\right)^{2} .
$$


Proof. For any $\mathbf{g} \in L^{2}(\Omega)^{d}$, we have

$$
\begin{aligned}
\mathcal{J} & :=\sum_{\mathbf{i}} \int_{\Pi_{\mathbf{i}}^{\varepsilon}} \mathbf{g}(\mathbf{x}) \cdot \boldsymbol{\eta}\left(\frac{\mathbf{x}-\mathbf{x}_{\mathbf{i}}}{\varepsilon}\right) \mathrm{d} \mathbf{x}=\sum_{k=1}^{d} \sum_{\mathbf{i}} \int_{\Pi_{\mathbf{i}}^{\varepsilon}} g_{k}(\mathbf{x}) \eta_{k}\left(\frac{\mathbf{x}-\mathbf{x}_{\mathbf{i}}}{\varepsilon}\right) \mathrm{d} \mathbf{x} \\
& =\sum_{k=1}^{d} \sum_{\mathbf{i}} \int_{\Pi_{\mathbf{i}}^{\varepsilon}}\left(g_{k}(\mathbf{x})-\left\langle g_{k}\right\rangle_{\Pi_{\mathbf{i}}^{\varepsilon}}\right) \eta_{k}\left(\frac{\mathbf{x}-\mathbf{x}_{\mathbf{i}}}{\varepsilon}\right) \mathrm{d} \mathbf{x}+\sum_{k=1}^{d} \sum_{\mathbf{i}} \int_{\Pi_{\mathbf{i}}^{\varepsilon}}\left\langle g_{k}\right\rangle_{\Pi_{\mathbf{i}}^{\varepsilon}} \eta_{k}\left(\frac{\mathbf{x}-\mathbf{x}_{\mathbf{i}}}{\varepsilon}\right) \mathrm{d} \mathbf{x} .
\end{aligned}
$$

Since

$$
\begin{aligned}
\sum_{\mathbf{i}} \int_{\Pi_{\mathbf{i}}^{\varepsilon}}\left\langle g_{k}\right\rangle_{\Pi_{\mathbf{i}}^{\varepsilon}} \eta_{k}\left(\frac{\mathbf{x}-\mathbf{x}_{\mathbf{i}}}{\varepsilon}\right) \mathrm{d} \mathbf{x} & =\varepsilon^{d}\left(\int_{\widehat{\Pi}} \eta_{k}(\mathbf{y}) \mathbf{d y}\right) \sum_{\mathbf{i}} \frac{1}{\left|\Pi_{\mathbf{i}}^{\varepsilon}\right|} \int_{\Pi_{\mathbf{i}}^{\varepsilon}} g_{k}(\mathbf{x}) \mathrm{d} \mathbf{x} \\
& =\varepsilon^{d}\left(\int_{\widehat{\Pi}} \eta_{k}(\mathbf{y}) \mathbf{d y}\right) \sum_{\mathbf{i}} \frac{1}{\varepsilon^{d}|\widehat{\Pi}|} \int_{\Pi_{\mathbf{i}}^{\varepsilon}} g_{k}(\mathbf{x}) \mathrm{d} \mathbf{x} \\
& =\int_{\widehat{\Pi}} \eta_{k}(\mathbf{y}) \mathbf{d y} \frac{1}{|\widehat{\Pi}|} \int_{\Omega} g_{k}(\mathbf{x}) \mathrm{d} \mathbf{x}=|\Omega|\left\langle g_{k}\right\rangle_{\Omega}\left\langle\eta_{k}\right\rangle_{\widehat{\Pi}}
\end{aligned}
$$

and for any $\left(c_{k}\right)_{k=1}^{d} \in \mathbb{R}^{d}$

$$
\begin{aligned}
\sum_{\mathbf{i}} \int_{\Pi_{\mathbf{i}}^{\varepsilon}}\left(g_{k}(\mathbf{x})-\left\langle g_{k}\right\rangle_{\Pi_{\mathbf{i}}^{\varepsilon}}\right) \eta_{k}\left(\frac{\mathbf{x}-\mathbf{x}_{\mathbf{i}}}{\varepsilon}\right) \mathrm{d} \mathbf{x} & =\sum_{\mathbf{i}} \int_{\Pi_{\mathbf{i}}^{\varepsilon}}\left(g_{k}(\mathbf{x})-\left\langle g_{k}\right\rangle_{\Pi_{\mathbf{i}}^{\varepsilon}}\right)\left(\eta_{k}\left(\frac{\mathbf{x}-\mathbf{x}_{\mathbf{i}}}{\varepsilon}\right)-c_{k}\right) \mathrm{d} \mathbf{x} \\
& \leqslant\left(\sum_{\mathbf{i}}\left\|g_{k}-\left\langle g_{k}\right\rangle_{\Pi_{\mathbf{i}}^{\varepsilon}}\right\|_{\Pi_{\mathbf{i}}^{\varepsilon}}\right)\left(\int_{\Pi_{\mathbf{i}}^{\varepsilon}}\left(\eta_{k}\left(\frac{\mathbf{x}-\mathbf{x}_{\mathbf{i}}}{\varepsilon}\right)-c_{k}\right)^{2} \mathrm{~d} \mathbf{x}\right)^{1 / 2} \\
& =\left(\sum_{\mathbf{i}}\left\|g_{k}-\left\langle g_{k}\right\rangle_{\Pi_{\mathbf{i}}^{\varepsilon}}\right\|_{\Pi_{\mathbf{i}}^{\varepsilon}}\right) \varepsilon^{d / 2}\left\|\eta_{k}-c_{k}\right\|_{\widehat{\Pi}}
\end{aligned}
$$

we find that

$$
\begin{aligned}
\mathcal{J} & \leqslant \sum_{k}\left(|\Omega|\left\langle g_{k}\right\rangle_{\Omega}\left\langle\eta_{k}\right\rangle_{\widehat{\Pi}}+\left(\delta_{\Omega}^{\mathrm{pw}} g\right)_{k}\left\|\eta_{k}-c_{k}\right\|_{\overparen{\Pi}}\right) \\
& \leqslant \sum_{k}\left(|\Omega|\left\langle g_{k}\right\rangle_{\Omega}\left\langle\eta_{k}\right\rangle_{\widehat{\Pi}}+\frac{\lambda_{k}}{2}\left(\delta_{\Omega}^{\mathrm{pw}} g\right)_{k}^{2}+\frac{1}{2 \lambda_{k}}\left\|\eta_{k}-c_{k}\right\|_{\overparen{\Pi}}^{2}\right) \\
& =|\Omega|\langle\mathbf{g}\rangle_{\Omega} \cdot\langle\boldsymbol{\eta}\rangle_{\widehat{\Pi}}+\frac{1}{2} \boldsymbol{\lambda} \cdot\left(\delta_{\Omega}^{\mathrm{pw}} \mathbf{g}\right)^{2}+\frac{1}{2} \int_{\widehat{\Pi}} \sum_{k} \frac{1}{\lambda_{k}}\left(\eta_{k}-c_{k}\right)^{2} \mathbf{d y}
\end{aligned}
$$

for any arbitrary vector $\lambda \in \mathbb{R}_{>0}^{d}$. In particular, we set $c_{k}=\left\langle\eta_{k}\right\rangle_{\widehat{\Pi}}$, and obtain (2.1).

In order to present the main estimate in a transparent form, we introduce the function

$$
\mathbf{g}_{\tau_{0}}(\mathbf{x}):=A_{\varepsilon} \nabla w_{\varepsilon}^{1}-\boldsymbol{\tau}_{\mathbf{0}}
$$

where

$$
\boldsymbol{\tau}_{\mathbf{0}} \in H(\Omega, \operatorname{div}):=\left\{\boldsymbol{\theta} \in\left(L^{2}(\Omega)\right)^{d}, \operatorname{div} \boldsymbol{\vartheta} \in L^{2}(\Omega)\right\}
$$

and the quantity

$$
\begin{aligned}
\mathcal{F}\left(w_{\varepsilon}^{1} ; \boldsymbol{\tau}_{\mathbf{0}}, \boldsymbol{\eta}, \boldsymbol{\lambda}, s\right):= & \left\|\mathbf{g}_{\boldsymbol{\tau}_{0}}\right\|_{A_{\varepsilon}^{-1}}^{2}+2 \varepsilon^{s}|\Omega|\left\langle\mathbf{g}_{\boldsymbol{\tau}_{0}}\right\rangle_{\Omega} \cdot\langle\boldsymbol{\eta}\rangle_{\widehat{\Pi}} \\
& +\varepsilon^{s}\left(\boldsymbol{\lambda}^{-1} \cdot\left(\delta_{\overparen{\Pi}} \boldsymbol{\eta}\right)^{2}+\boldsymbol{\lambda} \cdot\left(\delta_{\Omega}^{\mathrm{pw}}\left(\mathbf{g}_{\boldsymbol{\tau}_{0}}\right)\right)^{2}\right)+c_{0} \varepsilon^{2 s}\|\boldsymbol{\eta}\|_{\widehat{A}^{-1}, \widehat{\Pi}}^{2}
\end{aligned}
$$

where $\lambda \in \mathbb{R}_{>0}^{d}, s \in \mathbb{R}_{>0}$, and

$$
\boldsymbol{\eta} \in H_{0}(\widehat{\Pi}, \operatorname{div}):=\left\{\boldsymbol{\theta} \in H(\widehat{\Pi}, \operatorname{div}),\langle\operatorname{div} \boldsymbol{\vartheta}\rangle_{\widehat{\Pi}}=0\right\} .
$$

Now, we can deduce the first (general) form of the majorant $\mathcal{M}_{\oplus}$. It is presented in Theorem 2.1 (see also [25]), which proof uses the technique developed in [19-27]. 
Theorem 2.1. Let the cell of periodicity $\widehat{\Pi}$ be convex and the conditions (1.1), (1.3), (1.9), (1.11), and (1.13) be satisfied. Then, for any $\boldsymbol{\lambda} \in \mathbb{R}_{>0}^{d}, s \in \mathbb{R}_{>0}, \boldsymbol{\tau}_{\mathbf{0}} \in H\left(\Omega\right.$, div) and $\boldsymbol{\eta} \in H_{0}(\widehat{\Pi}$, div $)$ we have the estimate

$$
\begin{aligned}
\left\|\nabla\left(u_{\varepsilon}-w_{\varepsilon}^{1}\right)\right\|_{A_{\varepsilon}} \leqslant \mathcal{M}_{\oplus}\left(w_{\varepsilon}^{1}, \boldsymbol{\tau}_{\mathbf{0}}, \boldsymbol{\eta}, \boldsymbol{\lambda}, s\right):= & \mathcal{F}^{1 / 2}\left(w_{\varepsilon}^{1} ; \boldsymbol{\tau}_{\mathbf{0}}, \boldsymbol{\eta}, \boldsymbol{\lambda}, s\right) \\
& +\widetilde{C}_{F_{\Omega}}\left\|\operatorname{div} \boldsymbol{\tau}_{0}+f\right\|+\varepsilon^{s} \widetilde{C}\|\operatorname{div} \boldsymbol{\eta}\|_{\overparen{\Pi}}
\end{aligned}
$$

where $\mathcal{F}, \widetilde{C}_{F_{\Omega}}$ and $\widetilde{C}$ are defined by (2.6) and (2.13), respectively.

Proof. For any $v, w \in H_{0}^{1}(\Omega)$ and $\boldsymbol{\tau} \in H(\Omega$, div), we have

$$
\int_{\Omega} A_{\varepsilon} \nabla\left(u_{\varepsilon}-v\right) \cdot \nabla w=\int_{\Omega}\left(-A_{\varepsilon} \nabla v \cdot \nabla w+f w\right)=\int_{\Omega}\left(\boldsymbol{\tau}-A_{\varepsilon} \nabla v\right) \cdot \nabla w+\int_{\Omega}(\operatorname{div} \boldsymbol{\tau}+f) w .
$$

We set $w=u_{\varepsilon}-v$ and estimate the first term in (2.9) as follows:

$$
\int_{\Omega}\left(\boldsymbol{\tau}-A_{\varepsilon} \nabla v\right) \cdot \nabla\left(u_{\varepsilon}-v\right) \leqslant\left\|\nabla\left(u_{\varepsilon}-v\right)\right\|_{A_{\varepsilon}}\left\|A_{\varepsilon} \nabla v-\boldsymbol{\tau}\right\|_{A_{\varepsilon}^{-1}}
$$

Henceforth, we select $\boldsymbol{\tau}$ in a special form, namely,

$$
\boldsymbol{\tau}(\mathbf{x})=\boldsymbol{\tau}_{0}(\mathbf{x})-\varepsilon^{s} \boldsymbol{\eta}\left(\frac{\mathbf{x}-\mathbf{x}_{\mathbf{i}}}{\varepsilon}\right) \text { on } \Pi_{\mathbf{i}}^{\varepsilon}
$$

where

$$
\boldsymbol{\eta} \in H_{0}(\widehat{\Pi}, \operatorname{div}) .
$$

Since

$$
\operatorname{div} \boldsymbol{\tau}(\mathbf{x})=\operatorname{div} \boldsymbol{\tau}_{0}(\mathbf{x})-\varepsilon^{s} \operatorname{div} \boldsymbol{\eta}\left(\frac{\mathbf{x}-\mathbf{x}_{\mathbf{i}}}{\varepsilon}\right) \quad \forall \mathbf{x} \in \Pi_{\mathbf{i}}^{\varepsilon}, \forall \mathbf{i}
$$

and

$$
\left\langle\operatorname{div} \boldsymbol{\eta}\left(\frac{\cdot-\mathbf{x}_{\mathbf{i}}}{\varepsilon}\right)\right\rangle_{\Pi_{\mathbf{i}}^{\varepsilon}}=\varepsilon^{d-1}\langle\operatorname{div} \boldsymbol{\eta}\rangle_{\widehat{\Pi}}=0
$$

we obtain

$$
\begin{aligned}
\int_{\Omega}(\operatorname{div} \boldsymbol{\tau}+f)\left(u_{\varepsilon}-v\right) \mathrm{d} \mathbf{x} & =\int_{\Omega}\left(\operatorname{div} \boldsymbol{\tau}_{0}+f\right)\left(u_{\varepsilon}-v\right) \mathrm{d} \mathbf{x}-\sum_{\mathbf{i}} \int_{\Pi_{\mathbf{i}}^{\varepsilon}} \varepsilon^{s} \operatorname{div} \boldsymbol{\eta}\left(\frac{\cdot-\mathbf{x}_{\mathbf{i}}}{\varepsilon}\right)\left(u_{\varepsilon}-v\right) \mathrm{d} \mathbf{x} \\
& \leqslant C_{F_{\Omega}}\left\|\operatorname{div} \boldsymbol{\tau}_{0}+f\right\|\left\|\nabla\left(u_{\varepsilon}-v\right)\right\|+\varepsilon^{s} \sum_{\mathbf{i}} \varepsilon^{d / 2-1}\|\operatorname{div} \boldsymbol{\eta}\|_{\widehat{\Pi}} C_{\Pi_{\mathbf{i}}^{\varepsilon}}\left\|\nabla\left(u_{\varepsilon}-v\right)\right\|_{\Pi_{\mathbf{i}}^{\varepsilon}}
\end{aligned}
$$

where $C_{F_{\Omega}}$ is a constant in the Friedrich's inequality for $\Omega$ and $C_{\Pi_{\mathrm{i}}^{\varepsilon}}$ is a constant in the Poincare's inequality for $\Pi_{\mathbf{i}}^{\varepsilon}$. It is known (cf. [18]) that for convex $\Pi_{\mathbf{i}}^{\varepsilon}$

$$
C_{\Pi_{\mathbf{i}}^{\varepsilon}} \leqslant \frac{\operatorname{diam} \Pi_{\mathbf{i}}^{\varepsilon}}{\pi} \quad \forall d \geqslant 1 .
$$

We use (1.1) and (1.2) and arrive at the estimate

$$
\begin{aligned}
\int_{\Omega}(\operatorname{div} \boldsymbol{\tau}+f)\left(u_{\varepsilon}-v\right) \mathrm{d} \mathbf{x} & \leqslant C_{F_{\Omega}}\left\|\operatorname{div} \boldsymbol{\tau}_{0}+f\right\|\left\|\nabla\left(u_{\varepsilon}-v\right)\right\|+\varepsilon^{s} \varepsilon^{d / 2-1}\|\operatorname{div} \boldsymbol{\eta}\|_{\widehat{\Pi}} \sqrt{c_{0}} \varepsilon^{-d / 2} \varepsilon \frac{\varrho}{\pi}\left\|\nabla\left(u_{\varepsilon}-v\right)\right\| \\
& =C_{F_{\Omega}}\left\|\operatorname{div} \boldsymbol{\tau}_{0}+f\right\|\left\|\nabla\left(u_{\varepsilon}-v\right)\right\|+\varepsilon^{s} \frac{\varrho}{\pi} \sqrt{c_{0}}\|\operatorname{div} \boldsymbol{\eta}\|_{\widehat{\Pi}}\left\|\nabla\left(u_{\varepsilon}-v\right)\right\| .
\end{aligned}
$$

In view of (1.3), we obtain

$$
\int_{\Omega}(\operatorname{div} \boldsymbol{\tau}+f)\left(u_{\varepsilon}-v\right) \leqslant \widetilde{C}_{F_{\Omega}}\left\|\operatorname{div} \boldsymbol{\tau}_{0}+f\right\|\left\|\nabla\left(u_{\varepsilon}-v\right)\right\|_{A_{\varepsilon}}+\varepsilon^{s} \widetilde{C}\|\operatorname{div} \boldsymbol{\eta}\|_{\widehat{\Pi}}\left\|\nabla\left(u_{\varepsilon}-v\right)\right\|_{A_{\varepsilon}}
$$

where

$$
\tilde{C}_{F_{\Omega}}:=\frac{C_{F_{\Omega}}}{\sqrt{c_{1}}}, \quad \tilde{C}:=\frac{\varrho}{\pi} \sqrt{\frac{c_{0}}{c_{1}}} .
$$

Now (2.9), (2.10), and (2.12) imply the estimate

$$
\left\|\nabla\left(u_{\varepsilon}-v\right)\right\|_{A_{\varepsilon}} \leqslant\left\|A_{\varepsilon} \nabla v-\boldsymbol{\tau}_{0}+\varepsilon^{s} \boldsymbol{\eta}\right\|_{A_{\varepsilon}^{-1}}+\widetilde{C}_{F_{\Omega}}\left\|\operatorname{div} \boldsymbol{\tau}_{0}+f\right\|+\varepsilon^{s} \widetilde{C}\|\operatorname{div} \boldsymbol{\eta}\|_{\bar{\Pi}} .
$$


Consider the first term in the right-hand side of the estimate (2.14). We have

$$
\begin{aligned}
\left\|A_{\varepsilon} \nabla v-\boldsymbol{\tau}_{0}+\varepsilon^{s} \boldsymbol{\eta}\right\|_{A_{\varepsilon}^{-1}}^{2}= & \sum_{\mathbf{i}} \int_{\Pi_{\mathbf{i}}^{\varepsilon}} \widehat{A}^{-1}\left(\frac{\mathbf{x}-\mathbf{x}_{\mathbf{i}}}{\varepsilon}\right)\left(\widehat{A}\left(\frac{\mathbf{x}-\mathbf{x}_{\mathbf{i}}}{\varepsilon}\right) \nabla v(\mathbf{x})-\boldsymbol{\tau}_{0}(\mathbf{x})+\varepsilon^{s} \boldsymbol{\eta}\left(\frac{\mathbf{x}-\mathbf{x}_{\mathbf{i}}}{\varepsilon}\right)\right) \\
& \times\left(\widehat{A}\left(\frac{\mathbf{x}-\mathbf{x}_{\mathbf{i}}}{\varepsilon}\right) \nabla v(\mathbf{x})-\boldsymbol{\tau}_{0}(\mathbf{x})+\varepsilon^{s} \boldsymbol{\eta}\left(\frac{\mathbf{x}-\mathbf{x}_{\mathbf{i}}}{\varepsilon}\right)\right) \mathrm{d} \mathbf{x} .
\end{aligned}
$$

We set $v=w_{\varepsilon}^{1}$ and obtain with the help of (2.4)

$$
\begin{aligned}
\left\|A_{\varepsilon} \nabla w_{\varepsilon}^{1}-\boldsymbol{\tau}_{0}+\varepsilon^{s} \boldsymbol{\eta}\right\|_{A_{\varepsilon}^{-1}}^{2}= & \sum_{\mathbf{i}} \int_{\Pi_{\mathbf{i}}^{\varepsilon}}\left(\varepsilon^{2 s} \widehat{A}^{-1}\left(\frac{\mathbf{x}-\mathbf{x}_{\mathbf{i}}}{\varepsilon}\right) \boldsymbol{\eta}\left(\frac{\mathbf{x}-\mathbf{x}_{\mathbf{i}}}{\varepsilon}\right) \cdot \boldsymbol{\eta}\left(\frac{\mathbf{x}-\mathbf{x}_{\mathbf{i}}}{\varepsilon}\right)\right. \\
& \left.+2 \widehat{A}^{-1}\left(\frac{\mathbf{x}-\mathbf{x}_{\mathbf{i}}}{\varepsilon}\right) \varepsilon^{s} \mathbf{g}_{\tau_{0}}(\mathbf{x}) \cdot \boldsymbol{\eta}\left(\frac{\mathbf{x}-\mathbf{x}_{\mathbf{i}}}{\varepsilon}\right)+\widehat{A}^{-1}\left(\frac{\mathbf{x}-\mathbf{x}_{\mathbf{i}}}{\varepsilon}\right) \mathbf{g}_{\tau_{0}}(\mathbf{x}) \cdot \mathbf{g}_{\tau_{0}}(\mathbf{x})\right) \mathrm{d} \mathbf{x} .
\end{aligned}
$$

Now we apply Lemma 2.1 to the second term in the right-hand side of the above relation and arrive at (2.8).

We note that the estimate (2.14) also holds in a more general setting and can be applied to any reconstruction $v$ (including numerical one) of $u_{\varepsilon}$ with the requirement that $v \in H^{1}(\Omega)$.

Remark 2.1. It is not difficult to show that the majorant has the same convergence rate as the a priori estimate (cf. (1.16)) provided that the parameters are selected as is recommended by the theory [14].

Indeed, let us choose

$$
\tau_{\mathbf{0}}:=\mathbf{v}_{\mathbf{0}}-\varepsilon \mathbf{v}_{\mathbf{1}}^{\star}
$$

where $\mathbf{v}_{\mathbf{0}}$ and $\mathbf{v}_{\mathbf{1}}$ are defined by (1.17) and $\star$ means periodification of a function, i.e.

$$
\mathbf{w}^{\star}(\mathbf{x}):=\mathbf{w}\left(\mathbf{x}, \frac{\mathbf{x}-\mathbf{x}_{\mathbf{i}}}{\varepsilon}\right)
$$

for any $\mathbf{x} \in \Pi_{\mathbf{i}}^{\varepsilon}$ and for any $\mathbf{i}$. Then,

$$
\operatorname{div} \boldsymbol{\tau}_{0}=\operatorname{div} \mathbf{v}_{\mathbf{0}}-\varepsilon \operatorname{div} \mathbf{v}^{\star}{ }_{1}=\operatorname{div} \mathbf{v}_{0}-\varepsilon\left(\left(\operatorname{div}_{\mathbf{x}} \mathbf{v}_{1}\right)^{\star}+\varepsilon^{-1}\left(\operatorname{div}_{\mathbf{y}} \mathbf{v}_{1}\right)^{\star}\right) .
$$

Since $\mathbf{v}_{\mathbf{1}}:=-\operatorname{curl}_{\mathbf{x}}(\widetilde{N} \boldsymbol{\mu})$, (cf. (1.17)), the first term in the brackets vanishes and for the second one we use the fact that

$$
\left(\operatorname{div}_{\mathbf{y}} \mathbf{v}_{\mathbf{1}}\right)^{\star}=f+\operatorname{div}_{\mathbf{x}} \mathbf{v}_{\mathbf{0}}
$$

(see, e.g., [7], p. 65). Then, we obtain

$$
\operatorname{div} \boldsymbol{\tau}_{0}=\operatorname{div} \mathbf{v}_{\mathbf{0}}-f-\operatorname{div} \mathbf{v}_{\mathbf{0}}=-f .
$$

Therefore,

$$
\left\|\nabla\left(u_{\varepsilon}-w_{\varepsilon}^{1}\right)\right\|_{A_{\varepsilon}} \leqslant \mathcal{M}_{\oplus}\left(w_{\varepsilon}^{1}, \boldsymbol{\tau}_{0}, \boldsymbol{\eta}, \boldsymbol{\lambda}, s\right)=\mathcal{F}^{1 / 2}\left(w_{\varepsilon}^{1} ; \boldsymbol{\tau}_{0}, \boldsymbol{\eta}, \boldsymbol{\lambda}, s\right)+\varepsilon^{s} \widetilde{C}\|\operatorname{div} \boldsymbol{\eta}\|_{\widehat{\Pi}}
$$

where $\mathcal{F}$ is defined by (2.6) and

$$
\mathbf{g}_{\boldsymbol{\tau}_{0}}(\mathbf{x})=A_{\varepsilon} \nabla w_{\varepsilon}^{1}-\left(\mathbf{v}_{\mathbf{0}}-\varepsilon \mathbf{v}_{\mathbf{1}}\right) .
$$

Then, with the help of (1.15), (1.16), and the triangle inequality, we find that

$$
\begin{aligned}
\left\|\mathbf{g}_{\tau_{0}}(\mathbf{x})\right\|_{A_{\varepsilon}^{-1}} & =\left\|A_{\varepsilon} \nabla\left(w_{\varepsilon}^{1}-u_{\varepsilon}+u_{\varepsilon}\right)-\left(\mathbf{v}_{\mathbf{0}}-\varepsilon \mathbf{v}_{\mathbf{1}}\right)\right\|_{A_{\varepsilon}^{-1}} \\
& \leqslant\left\|A_{\varepsilon} \nabla\left(w_{\varepsilon}^{1}-u_{\varepsilon}\right)\right\|_{A_{\varepsilon}^{-1}}+\left\|A_{\varepsilon} \nabla u_{\varepsilon}-\left(\mathbf{v}_{\mathbf{0}}-\varepsilon \mathbf{v}_{\mathbf{1}}\right)\right\|_{A_{\varepsilon}^{-1}} \leqslant c \sqrt{\varepsilon} .
\end{aligned}
$$

We set $\boldsymbol{\eta}=\mathbf{0}$, tend all components of $\lambda$ to zero and find that

$$
\mathcal{M}_{\oplus} \leqslant c \varepsilon^{1 / 2} .
$$

It is worth noting that in some special cases this asymptotic result can be proved in a simpler way. For example, if

$$
A_{0}=\left\langle\widehat{A}^{-1}\right\rangle_{\widehat{\Pi}}^{-1}
$$


(which is always the situation in the one-dimensional case or if $\operatorname{curl} \widehat{A}^{-1}=\mathbf{0}$ ), then the simplest choice

$$
\boldsymbol{\tau}_{0}=A_{0} \nabla u_{0}
$$

implies $\operatorname{div} \boldsymbol{\tau}_{0}=-f$. In this case,

$$
\left\|\nabla\left(u_{\varepsilon}-w_{\varepsilon}^{1}\right)\right\|_{A_{\varepsilon}} \leqslant \mathcal{M}_{\oplus}\left(w_{\varepsilon}^{1}, \boldsymbol{\tau}_{0}, \boldsymbol{\eta}, \boldsymbol{\lambda}, s\right):=\mathcal{F}^{1 / 2}\left(w_{\varepsilon}^{1} ; \boldsymbol{\tau}_{0}, \boldsymbol{\eta}, \boldsymbol{\lambda}, s\right)+\varepsilon^{s} \widetilde{C}\|\operatorname{div} \boldsymbol{\eta}\|_{\widehat{\Pi}}
$$

where $\mathcal{F}$ is defined by $(2.6)$ and

$$
\mathbf{g}_{\tau_{0}}(\mathbf{x})=A_{\varepsilon} \nabla w_{\varepsilon}^{1}-A_{0} \nabla u_{0}
$$

for all $\mathbf{y} \in \widehat{\Pi}, \mathbf{x} \in \Pi_{\mathbf{i}}^{\varepsilon}$. Choosing again $\eta=\mathbf{0}$ in (2.19), we obtain (2.18).

Remark 2.2. The right-hand side of the majorant (2.8) is the sum of three non-negative terms, which include a global function $\boldsymbol{\tau}_{0}$ and a function $\boldsymbol{\eta}$ defined on the cell of periodicity. This reflects the specifics of the considered class of problems. Hence, the computation of the majorant is based on the flux of the homogenized solution and a proper selection (cf. Section 4) of the function $\boldsymbol{\eta}$ defined on the cell of periodicity. The scalar parameters $\lambda_{i}$ and the power $s$ can be selected in order to minimize the overall value of the majorant. We emphasize that the computation of the majorant does not require an approximation of the flux associated with the original (global) periodic problem.

The choice

$$
\tau_{0}=A_{0} \nabla u_{0}, \quad \eta=\mathbf{0}
$$

leads to the simplified error estimator

$$
\begin{aligned}
\left\|\nabla\left(u_{\varepsilon}-w_{\varepsilon}^{1}\right)\right\|_{A_{\varepsilon}} & \leqslant\left|\sum_{\mathbf{i}} \int_{\Pi_{\mathbf{i}}^{\varepsilon}} \widehat{A}^{-1}\left(\frac{\mathbf{x}-\mathbf{x}_{\mathbf{i}}}{\varepsilon}\right) \mathbf{g}_{\tau_{0}}(\mathbf{x}) \cdot \mathbf{g}_{\boldsymbol{\tau}_{0}}(\mathbf{x}) \mathrm{d} \mathbf{x}\right|^{1 / 2} \\
& =\left\|A_{\varepsilon} \nabla w_{\varepsilon}^{1}-A_{0} \nabla u_{0}\right\|_{A_{\varepsilon}^{-1}=: M_{\oplus}\left(w_{\varepsilon}^{1}, u_{0}\right) .}
\end{aligned}
$$

It is easy to show that this simplified majorant is equivalent to the combined primal-dual norm

$$
\left[u_{\varepsilon}-w_{\varepsilon}^{1}, A_{\varepsilon} \nabla u_{\varepsilon}-\tau_{0}\right]:=\left\|\nabla\left(u_{\varepsilon}-w_{\varepsilon}^{1}\right)\right\|_{A_{\varepsilon}}+\left\|A_{\varepsilon} \nabla u_{\varepsilon}-\tau_{0}\right\|_{A_{\varepsilon}^{-1}}
$$

Indeed, from one hand

$$
\begin{aligned}
{\left[u_{\varepsilon}-w_{\varepsilon}^{1}, A_{\varepsilon} \nabla u_{\varepsilon}-\tau_{0}\right] } & =\left\|\nabla\left(u_{\varepsilon}-w_{\varepsilon}^{1}\right)\right\|_{A_{\varepsilon}}+\left\|A_{\varepsilon} \nabla u_{\varepsilon}-A_{0} \nabla u_{0}\right\|_{A_{\varepsilon}^{-1}} \\
& \leqslant\left\|\nabla\left(u_{\varepsilon}-w_{\varepsilon}^{1}\right)\right\|_{A_{\varepsilon}}+\left\|A_{\varepsilon} \nabla u_{\varepsilon}-A_{\varepsilon} \nabla w_{\varepsilon}^{1}\right\|_{A_{\varepsilon}^{-1}}+\left\|A_{\varepsilon} \nabla w_{\varepsilon}^{1}-A_{0} \nabla u_{0}\right\|_{A_{\varepsilon}^{-1}} \\
& \leqslant 3\left\|A_{\varepsilon} \nabla u_{\varepsilon}-A_{0} \nabla u_{0}\right\|_{A_{\varepsilon}^{-1}}=3 M_{\oplus}\left(w_{\varepsilon}^{1}, u_{0}\right) .
\end{aligned}
$$

From the other hand,

$$
\begin{aligned}
M_{\oplus}\left(w_{\varepsilon}^{1}, u_{0}\right)=\left\|A_{\varepsilon} \nabla w_{\varepsilon}^{1}-\tau_{0}\right\|_{A_{\varepsilon}^{-1}} & \leqslant\left\|A_{\varepsilon} \nabla w_{\varepsilon}^{1}-A_{\varepsilon} \nabla u_{\varepsilon}\right\|_{A_{\varepsilon}^{-1}}+\left\|A_{\varepsilon} \nabla u_{\varepsilon}-\tau_{0}\right\|_{A_{\varepsilon}^{-1}} \\
& =\left[u_{\varepsilon}-w_{\varepsilon}^{1}, A_{\varepsilon} \nabla u_{\varepsilon}-\tau_{0}\right] .
\end{aligned}
$$

Hence, we obtain

$$
M_{\oplus}\left(w_{\varepsilon}^{1}, u_{0}\right) \leqslant\left[u_{\varepsilon}-w_{\varepsilon}^{1}, A_{\varepsilon} \nabla u_{\varepsilon}-\tau_{0}\right] \leqslant 3 M_{\oplus}\left(w_{\varepsilon}^{1}, u_{0}\right)
$$

We note that this result is similar to that has been obtained in [22] for errors of mixed approximations of elliptic partial differential equations.

Remark 2.3. One can show that in the one-dimensional case, (2.20) holds as equality provided that

$$
\int_{\Omega}\left(A_{\varepsilon}^{-1} \int_{0}^{x} f\right)=\int_{\Omega}\left(A_{0}^{-1} \int_{0}^{x} f\right) .
$$


Remark 2.4. In certain cases, we may know only numerical approximations to the solutions $N_{k}, \widetilde{N}_{k}$ and $u_{0}$ of the auxiliary cell problems (cf. (1.8), (1.18)) and of the homogenized equation (cf. (1.10)). The corresponding approximation errors can be estimated by error majorants of similar types (see [19-24] and references therein). Then, the overall error majorant will include both, approximation and modeling errors. A combined modeling-discretization strategy is suggested in [24] (where the modeling error is generated by defeaturing of a complicated structure) and in [28] (where the modeling error is generated by dimension reduction) and should be used in this case. This topic deserves a separate investigation and lies beyond the framework of this paper which is focused on the principal structure of the guaranteed error bound for homogenized problems.

\section{Lower bound of the modeling error}

Lower bounds of the modeling error allows us to estimate numerically the sharpness of the error majorant and to evaluate the efficiency of error estimation. A lower bound of the energy error norm can be derived by means of the well known relation (see, e.g., [22], pp. 85-86):

$$
\left\|\nabla\left(u_{\varepsilon}-v\right)\right\|_{A_{\varepsilon}}^{2}=\sup _{w \in H_{0}^{1}(\Omega)} \mathcal{M}_{\ominus}^{2}(v ; w):=\sup _{w \in H_{0}^{1}(\Omega)} \int_{\Omega}\left(2\left(f w-A_{\varepsilon} \nabla v \cdot \nabla w\right)-A_{\varepsilon} \nabla w \cdot \nabla w\right) .
$$

Clearly, for any $w \in H_{0}^{1}(\Omega)$ it holds $\left\|\nabla\left(u_{\varepsilon}-v\right)\right\|_{A_{\varepsilon}} \geqslant \mathcal{M}_{\ominus}(v ; w)$. Moreover, there exists a function $w$ such that the inequality holds as equality. We use (3.1) with $v=w_{\varepsilon}^{1}$ (cf. (1.11)) and represent $w$ in the form $w=\rho_{\max } z$, where $z \in H_{0}^{1}(\Omega)$ is a certain specially selected function and the multiplier $\rho_{\max }$ is defined by the relation

$$
\rho_{\max }=\frac{\int_{\Omega}\left(f z-A_{\varepsilon} \nabla w_{\varepsilon}^{1} \cdot \nabla z\right)}{\int_{\Omega} A_{\varepsilon} \nabla z \cdot \nabla z} .
$$

In this case, $\mathcal{M}_{\ominus}^{2}\left(w_{\varepsilon}^{1} ; \rho z\right)$ attains its maximum as a quadratic function with respect to $\rho$. Inserting this value into $\mathcal{M}_{\ominus}^{2}(v ; w)$, we obtain the following lower bound of the modeling error $\left\|\nabla\left(u_{\varepsilon}-w_{\varepsilon}^{1}\right)\right\|_{A_{\varepsilon}}$ :

$$
\mathcal{M}_{\ominus}\left(w_{\varepsilon}^{1} ; z\right):=\frac{\left|\int_{\Omega}\left(f z-A_{\varepsilon} \nabla w_{\varepsilon}^{1} \cdot \nabla z\right)\right|}{\|\nabla z\|_{A_{\varepsilon}}}=\frac{\left|\int_{\Omega}\left(A_{0} \nabla u_{0}-A_{\varepsilon} \nabla w_{\varepsilon}^{1}\right) \cdot \nabla z\right|}{\|\nabla z\|_{A_{\varepsilon}}} .
$$

Below we consider two possible choices of the function $z$. Let

$$
z(\mathbf{x}):=w_{\varepsilon}^{1}(\mathbf{x})-u_{0}(\mathbf{x})-\varepsilon \Theta\left(\frac{\mathbf{x}-\mathbf{x}_{\mathbf{i}}}{\varepsilon}\right)
$$

where $\Theta\left(\left(\mathbf{x}-\mathbf{x}_{\mathbf{i}}\right) / \varepsilon\right)$ is a periodic function defined in $\widehat{\Pi}$, and

$$
\varphi_{\varepsilon}^{0}\left(\mathbf{x}, \frac{\mathbf{x}-\mathbf{x}_{\mathbf{i}}}{\varepsilon}\right):=-\psi^{\varepsilon}(\mathbf{x}) \boldsymbol{N}\left(\frac{\mathbf{x}-\mathbf{x}_{\mathbf{i}}}{\varepsilon}\right) \cdot \nabla u_{0}(\mathbf{x}) .
$$

Then, we rewrite (1.11) in the form

$$
w_{\varepsilon}^{1}=u_{0}+\varepsilon \varphi_{\varepsilon}^{0}
$$

and

$$
z(\mathbf{x})=\varepsilon\left(\varphi_{\varepsilon}^{0}\left(\mathbf{x}, \frac{\mathbf{x}-\mathbf{x}_{\mathbf{i}}}{\varepsilon}\right)-\Theta\left(\frac{\mathbf{x}-\mathbf{x}_{\mathbf{i}}}{\varepsilon}\right)\right)
$$

we see that in this case the test function $z$ is a periodical function. The minorant is defined by the relation

$$
\mathcal{M}_{\Theta}^{2}\left(w_{\varepsilon}^{1}, \Theta\right)=\frac{\left(\int_{\Omega}\left[\mathbf{q} \cdot \nabla \varphi_{\varepsilon}^{0}-\mathbf{q} \cdot \nabla \Theta\right]\right)^{2}}{\int_{\Omega} A_{\varepsilon} \nabla\left(\varphi_{\varepsilon}^{0}-\Theta\right) \cdot \nabla\left(\varphi_{\varepsilon}^{0}-\Theta\right)}
$$

where

$$
\mathbf{q}:=\left(A_{0}-A_{\varepsilon}\right) \nabla u_{0}-\varepsilon A_{\varepsilon} \nabla \varphi_{\varepsilon}^{0} .
$$


For this ansatz, the best lower bound will be obtained if (3.7) is maximized with respect to the cell based function $\Theta$ and global function $\psi_{\varepsilon}$. However, in general, finding these (optimal) functions may require essential computational efforts. In the tests below, we used a much simpler choice, namely,

$$
\Theta=0, \quad \psi_{\varepsilon}=\min \left\{1, \frac{1}{\varepsilon} \operatorname{dist}(\mathbf{x}, \partial \Omega)\right\}
$$

and the minorant (3.7) is reduced to

$$
\mathcal{M}_{\ominus}^{\mathrm{per}}\left(w_{\varepsilon}^{1} ; \mathbf{0}\right)=\frac{\left|\int_{\Omega} \mathbf{q} \cdot \nabla \varphi_{\varepsilon}^{0}\right|}{\left\|\nabla \varphi_{\varepsilon}^{0}\right\|_{A_{\varepsilon}}} .
$$

Also, we may try to find a suitable $z$ represented aperiodically, for example in the form $u_{0}$ plus small quasi-periodical disturbances

$$
z(\mathbf{x})=\rho\left(u_{0}(\mathbf{x})+\varepsilon \psi^{\varepsilon} \Theta\left(\frac{\mathbf{x}-\mathbf{x}_{\mathbf{i}}}{\varepsilon}\right)\right) .
$$

In this case,

$$
\mathcal{M}_{\Theta}^{2}\left(w_{\varepsilon}^{1}, \Theta\right)=\frac{\left(\int_{\Omega}\left[\widetilde{\mathbf{q}} \cdot \nabla u_{0}+\widetilde{\mathbf{q}} \cdot \nabla\left(\varepsilon \psi^{\varepsilon} \Theta\right)\right]\right)^{2}}{\int_{\Omega} A_{\varepsilon} \nabla\left(u_{0}+\varepsilon \psi^{\varepsilon} \Theta\right) \cdot \nabla\left(u_{0}+\varepsilon \psi^{\varepsilon} \Theta\right)},
$$

where

$$
\widetilde{\mathbf{q}}:=\left(A_{0}-A_{\varepsilon}\right) \nabla u_{0}+\varepsilon A_{\varepsilon} \nabla\left(\psi^{\varepsilon} \boldsymbol{N} \nabla u_{0}\right) .
$$

In general, the minorant should be maximized with respect to $\Theta$. However, even the simplest choice $\Theta=0$ yields a lower bound

$$
\mathcal{M}_{\ominus}^{\text {aper }}\left(w_{\varepsilon}^{1} ; \mathbf{0}\right)=\frac{\left|\int_{\Omega} \mathbf{q} \cdot \nabla u_{0}\right|}{\left\|\nabla u_{0}\right\|_{A_{\varepsilon}}}
$$

\section{Numerical experiments}

A general strategy of computing the majorant consists of minimizing $\mathcal{M}_{\oplus}$ with respect to parameters $\lambda, s$, vector function $\tau \in H\left(\Omega\right.$, div) and vector function $\eta \in H_{0}\left(\widehat{\Pi}\right.$, div) using finite dimensional subspaces $S_{h}(\Omega) \subset$ $H\left(\Omega\right.$, div) (e.g. a finite element space) and $S_{h}(\widehat{\Pi}) \subset H_{0}(\widehat{\Pi}$, div), respectively. The process can be started with

$$
\boldsymbol{\tau}_{0}=A_{0} \nabla u_{0}, \quad \boldsymbol{\eta}=0 .
$$

In the numerical experiments discussed below, we set $\boldsymbol{\tau}$ and $\boldsymbol{\eta}$ in accordance with (4.1) and use the simplest error estimator $M_{\oplus}\left(w_{\varepsilon}^{1}, u_{0}\right)$ :

$$
\left\|\nabla\left(u_{\varepsilon}-w_{\varepsilon}^{1}\right)\right\|_{A_{\varepsilon}} \leqslant\left|\sum_{\mathbf{i}} \int_{\Pi_{\mathbf{i}}^{\varepsilon}} \widehat{A}^{-1}\left(\frac{\mathbf{x}-\mathbf{x}_{\mathbf{i}}}{\varepsilon}\right) \mathbf{g}_{\tau_{0}}(\mathbf{x}) \cdot \mathbf{g}_{\tau_{0}}(\mathbf{x}) \mathrm{d} \mathbf{x}\right|^{1 / 2}
$$

where $\mathbf{g}_{\boldsymbol{\tau}_{0}}(\mathbf{x})$ is defined by (2.4). In most cases, this choice was enough in order to have sufficiently sharp estimates. This is explainable because if the periodic structure is fine and contains many cells, then the correction term is less significant and its influence can be diminished by increasing values of $s$. However, if a periodic structure is rather coarse (e.g., 25-50 cells) and/or the coefficients of the matrix $\widehat{A}$ have jumps, sharp oscillations, etc. then the term $\varepsilon^{s} \boldsymbol{\eta}$ may augment the homogenized flux substantially and it may be required to use the most general form of the majorant.

Below, we apply the estimates derived in Sections 2 and 3 to several one- and two-dimensional test problems. For this purpose, we select problems used in publications related to analysis of homogenized and interface problems, e.g., see $[8,13,15,16,30]$. Our goal is to validate the sharpness of the two-sided error bounds presented by $M_{\oplus}$ and two lower bounds introduced in Section 3 (i.e., $M_{\ominus}$ is computed by $\mathcal{M}_{\ominus}^{\text {per }}\left(w_{\varepsilon}^{1} ; \mathbf{0}\right)$ or $\mathcal{M}_{\ominus}^{\text {aper }}\left(w_{\varepsilon}^{1} ; \mathbf{0}\right)$; cf. (3.10) and (3.14)). 
For the quantitative characterization of two-sided bounds, we use the number

$$
\varkappa:=\frac{M_{\oplus}}{M_{\ominus}}
$$

which can be also viewed as a computable upper bound of the efficiency index

$$
i_{\oplus}^{\mathrm{eff}}:=\frac{M_{\oplus}}{\left\|\nabla\left(u_{\varepsilon}-w_{\varepsilon}^{1}\right)\right\|_{A_{\varepsilon}}}
$$

and gives insights of the quality of the error majorant. Similarly, we define the efficiency index of the lower bound

$$
i_{\ominus}^{\text {eff }}:=\frac{M_{\ominus}}{\left\|\nabla\left(u_{\varepsilon}-w_{\varepsilon}^{1}\right)\right\|_{A_{\varepsilon}}} .
$$

In the first series of tests, we set $d=1$ and $\Omega=(0,1)$. Then, $u_{\varepsilon} \in H_{0}^{1}(\Omega)$ is defined by the relation

$$
\int_{0}^{1} A_{\varepsilon} u_{\varepsilon}^{\prime} v^{\prime}=\int_{0}^{1} f v \quad \forall v \in H_{0}^{1}(\Omega) .
$$

Example 4.1. Let

$$
\widehat{A}(y):= \begin{cases}1, & 0<y \leqslant 1 / 2 \\ 2, & 1 / 2<y<1\end{cases}
$$

and $A_{\varepsilon}$ is defined as in (1.4). The right-hand side is given by $f:=\sin (2 \pi x / \varepsilon)$. Here, the explicit forms of $A_{0}$, $u_{0}^{\prime}, \mathrm{d} N / \mathrm{d} y$ and $N$ are known (they can be found from (1.9) and (1.8)):

$$
\begin{aligned}
& A_{0}(x)=\frac{4}{3} \\
& u_{0}^{\prime}=\frac{3 \varepsilon}{8 \pi} \cos \left(2 \pi x \varepsilon^{-1}\right) \\
& \frac{\mathrm{d} N}{\mathrm{~d} y}(y)= \begin{cases}-\frac{1}{3}, & 0<y \leqslant 1 / 2 \\
\frac{1}{3}, & 1 / 2<y<1\end{cases} \\
& N(y)= \begin{cases}-\frac{y}{3}+\frac{1}{12}, & 0<y \leqslant 1 / 2 \\
\frac{y}{3}-\frac{1}{4}, & 1 / 2<y<1 .\end{cases}
\end{aligned}
$$

Example 4.2. Let $A_{\varepsilon}(x)=2+\cos (2 \pi x / \varepsilon), f:=\mathrm{e}^{10 x}$. Then, see (1.9) and (1.8):

$$
\begin{aligned}
& A_{0}(x)=\sqrt{3} \\
& u_{0}^{\prime}=-\frac{3^{-0.5}}{10} \mathrm{e}^{10 x}+\frac{3^{-0.5}}{100} \mathrm{e}^{10} \\
& \frac{\mathrm{d} N}{\mathrm{~d} y}(y)=1-\sqrt{3}(2+\cos (2 \pi y))^{-1} \\
& N(y)=\int\left(1-\sqrt{3}(2+\cos (2 \pi y))^{-1}\right) \mathrm{d} y .
\end{aligned}
$$

In Example 4.1, $f$ is a periodic function. Therefore, it is natural to expect that the minorant $M_{\ominus}^{\text {per }}$ (in which the periodicity is taken into account) will provide better results. In Example 4.2, the right-hand side is represented by a non-periodical function, and, therefore, we expect that $M_{\ominus}^{\text {aper }}$ will be better (at least for problems with relatively small amount of cells). The corresponding numerical results are depicted in Fig. 2 and confirm the proposed choice of the lower error bound. We note that in Example 4.1 the equality (2.25) holds and (cf. Remark 2.3) the majorant (4.2) coincides with the error. This fact is confirmed numerically (see Fig. 2 a, b). Example 4.2 shows that the majorant and minorants are quite sharp if the number of cells is sufficiently large (regardless of the condition (2.25)). 


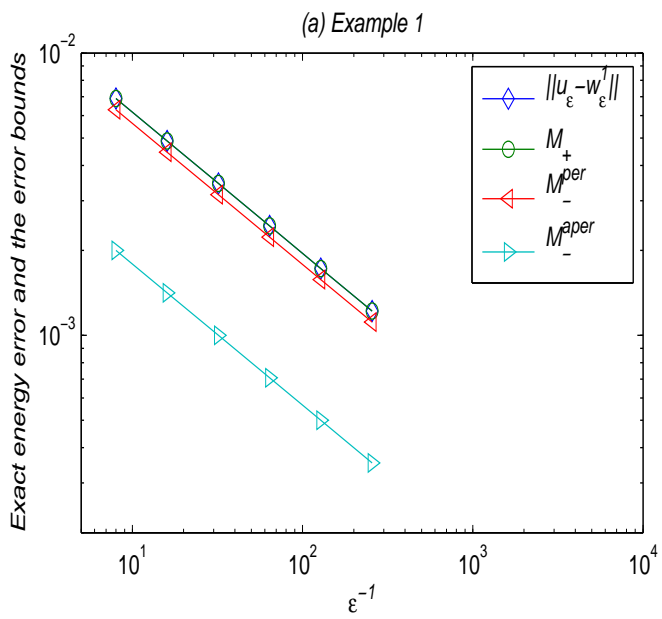

(b) Example 1
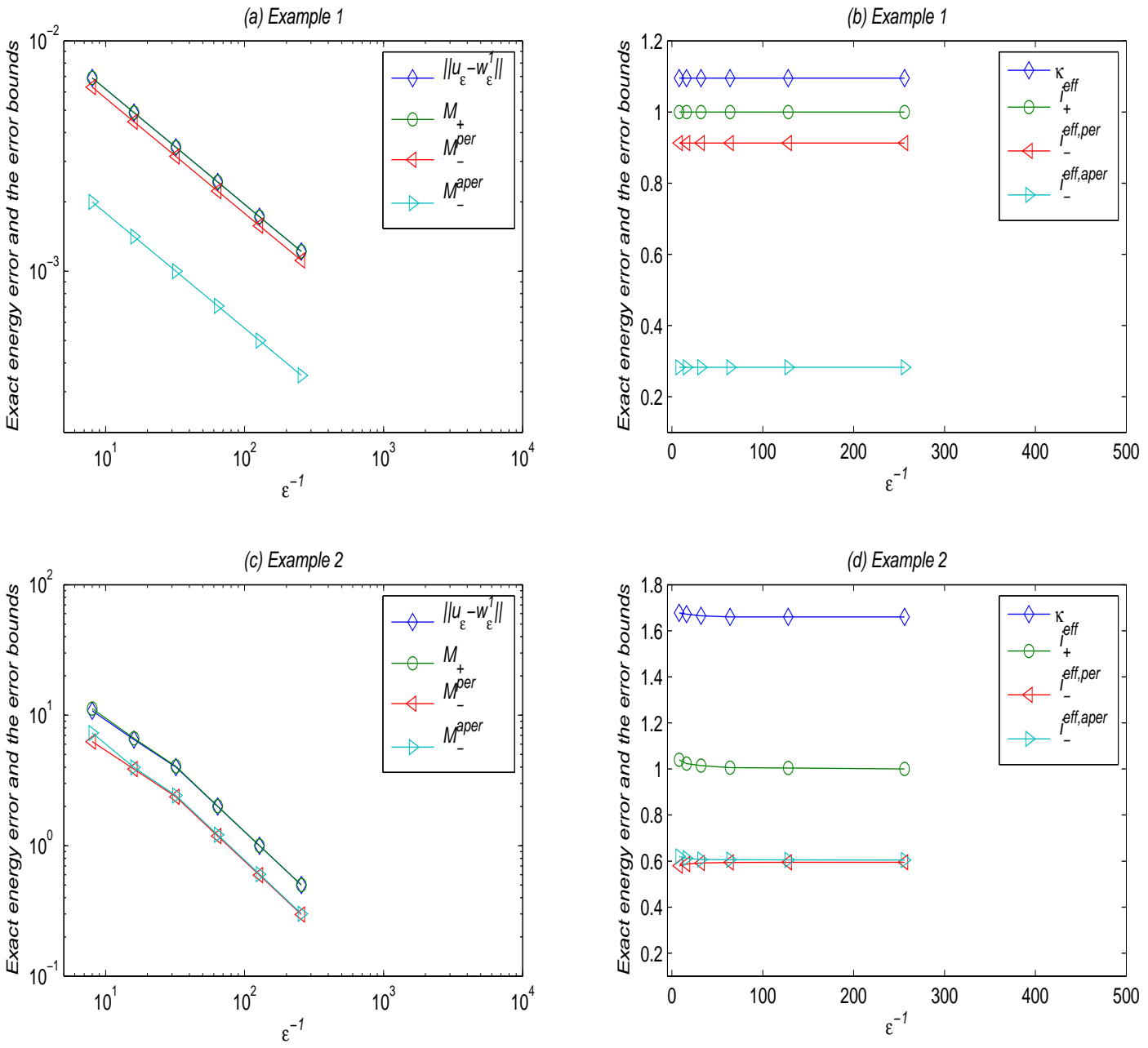

Figure 2. Error bounds (left) and efficiency indices (right) for Example 4.1 and Example 4.2.

Example 4.3. Let $d=2, \Omega=(0,1)^{2}$, and $u_{\varepsilon} \in H_{0}^{1}(\Omega)$ be defined by the relation

$$
\int_{\Omega} A_{\varepsilon} \nabla u_{\varepsilon} \cdot \nabla v=\int_{\Omega} f v \quad \forall v \in H_{0}^{1}(\Omega) .
$$

Here $A_{\varepsilon}$ is generated by the matrix $\widehat{A}:=a I$ (cf. (1.4)), where

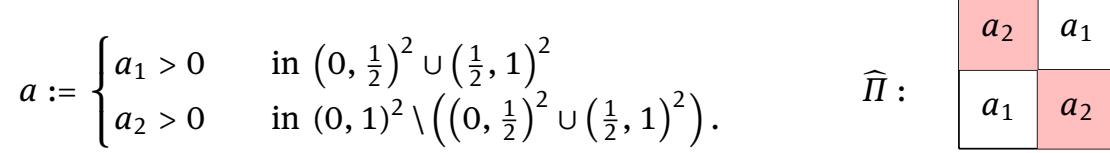

Then (see, e.g., [14], pp. 35-39), $A_{0}=\sqrt{a_{1} a_{2}}$. We choose

$$
f=2 \sqrt{a_{1} a_{2}}\left(x_{1}\left(1-x_{1}\right)+x_{2}\left(1-x_{2}\right)\right)
$$

such that

$$
u_{0}(\mathbf{x})=x_{1} x_{2}\left(1-x_{1}\right)\left(1-x_{2}\right) .
$$

Exact solutions of the cell problems

$$
\begin{gathered}
\frac{\partial}{\partial y_{i}}\left(\widehat{A}_{i j}(\mathbf{y}) \frac{\partial N_{k}(\mathbf{y})}{\partial y_{j}}\right)=\frac{\partial}{\partial y_{i}} \widehat{A}_{i k}(\mathbf{y}) \text { in } \widehat{\Pi}=(0,1)^{2} \\
N_{k} \text { is periodic in } \widehat{\Pi}
\end{gathered}
$$

$$
\left\langle N_{k}\right\rangle_{\widehat{\Pi}}=0
$$


are found in [15] in the form

$$
N_{k}(\mathbf{y})=v\left(\frac{\mathbf{y}+1}{2}\right)+y_{k}, \quad k=1,2 .
$$

Here $v(\mathbf{y})$ is the unique solution of the problem

$$
-\operatorname{div}(a \nabla v)=0 \quad \text { in }(-1,1)^{2}
$$

with homogenous Dirichlet boundary conditions and $a$ is defined by (4.5). This solution is given in polar coordinates $(r, \vartheta)$ centered at the origin by the relation

$$
v=r^{\gamma} \mu(\vartheta)
$$

where

$$
\mu(\vartheta):= \begin{cases}\cos (\alpha \beta) \cos \left(\left(\vartheta-\frac{\pi}{2}+\alpha\right) \gamma\right), & 0 \leqslant \vartheta \leqslant \frac{\pi}{2} \\ \cos (\alpha \gamma) \cos ((\vartheta-\pi+\beta) \gamma), & \frac{\pi}{2} \leqslant \vartheta \leqslant \pi \\ \cos (\alpha \beta) \cos ((\vartheta-\pi-\alpha) \gamma), & \pi \leqslant \vartheta \leqslant \frac{3 \pi}{2} \\ \cos \left(\left(\frac{\pi}{2}-\alpha\right) \gamma\right) \cos \left(\left(\vartheta-\frac{3 \pi}{2}-\beta\right) \gamma\right), & \frac{3 \pi}{2} \leqslant \vartheta \leqslant 2 \pi\end{cases}
$$

is a continuous and piecewise smooth function and the numbers $\alpha, \beta$, and $\gamma$ depend on $a_{1} / a_{2}$ and satisfy the relations

$$
\left\{\begin{array}{l}
\frac{a_{1}}{a_{2}}=\tan (\beta \gamma) \cot (\alpha \gamma) \\
\frac{a_{2}}{a_{1}}=-\tan (\alpha \gamma) \cot (\beta \gamma) \\
\frac{a_{1}}{a_{2}}=-\tan (\beta \gamma) \cot \left(\left(\frac{\pi}{2}-\alpha\right) \gamma\right) \\
\frac{a_{2}}{a_{1}}=\tan \left(\left(\frac{\pi}{2}-\alpha\right) \gamma\right) \cot (\beta \gamma) \\
\gamma>0 \\
\max \{0, \pi \gamma-\pi\}<2 \alpha \gamma<\min \{\pi \gamma, \pi\} \\
\max \{0, \pi\}<-2 \beta \gamma<\min \{\pi, 2 \pi\} .
\end{array}\right.
$$

It is known that $v$ has a restricted regularity (namely, $v \in H^{1+\gamma-\varepsilon}(\widehat{\Pi})$ for any $\varepsilon>0$ ).

We use this fact in order to verify the efficiency of the error majorant in different situations, we consider two cases, in which the ratio between $a_{1}$ and $a_{2}$ (and the regularity of $N_{k}$ ) are quite different.

- Case 1: let $a_{1}=5.0, a_{2}=1.0$. In this case, the solution (4.9) has $\gamma=0.53544094560$ and $\vartheta=\pi / 2$ (cf. system (3.2) in [15]) so that $v \in H^{3 / 2}(\Omega)$.

- Case 2: now, we set $\gamma=0.1$ and $\theta=\pi / 2$. By solving (4.11) and (4.12), we find that in this case $a_{1}=$ 161.4476387975881 and $a_{2}=1.0$. Here, $v \in H^{1+\alpha}(\widehat{\Pi})$ with $0<\alpha<0.1$, i.e., it is almost an $H^{1}$ function.

To quantify the efficiency of the estimates (4.2) and (3.10), we compare them with the exact error

$$
e:=\left\|\nabla\left(u_{\varepsilon}-w_{\varepsilon}^{1}\right)\right\|_{A_{\varepsilon}} .
$$

Since $u_{\varepsilon}$ is unknown, we replace it by the 'reference' solution $u_{\text {ref }}$ computed on a very fine mesh $(h \ll \varepsilon)$. The corresponding efficiency indices are defined by the relations

$$
i_{\oplus}^{\text {eff }}=\frac{M_{\oplus}\left(w_{\varepsilon}^{1} ; \mathbf{0}, \mathbf{1}, 1\right)}{\left\|\nabla\left(u_{\mathrm{ref}}-w_{\varepsilon}^{1}\right)\right\|_{A_{\varepsilon}}}, \quad i_{\ominus}^{\text {eff, per }}=\frac{M_{\ominus}^{\mathrm{per}}\left(w_{\varepsilon}^{1} ; \mathbf{0}\right)}{\left\|\nabla\left(u_{\mathrm{ref}}-w_{\varepsilon}^{1}\right)\right\|_{A_{\varepsilon}}} .
$$

In Table 1 (Case 1) and 2 (Case 2), we present these quantities together with the quantity $\varkappa$ as in (4.3). We see that the estimates adequately reproduce the modeling error.

It is quite predictable that the estimates are better in the first case (related to a more regular $v$ ). For the first problem, efficiency indices of the majorant and minorant are quite close to 1 . However, the estimates are also valid for the second case (minimal regularity). Indeed, the efficiency index of the majorant does not exceed 2.3 and the one of the minorant does not go below 0.7 . 
Table 1. Efficiency of error majorant and minorant for Example 4.3, Case 1.

\begin{tabular}{cccc}
\hline $\boldsymbol{\varepsilon}^{-1}$ & $\boldsymbol{i}_{\oplus}^{\text {eff }}$ & $\boldsymbol{i}_{\ominus}^{\text {eff }}$ & $\boldsymbol{\varkappa}$ \\
\hline 8 & 1.0714 & 0.8824 & 1.2141 \\
16 & 1.0874 & 0.8781 & 1.2384 \\
32 & 1.0988 & 0.8591 & 1.2790 \\
64 & 1.1633 & 0.8461 & 1.3749 \\
\hline
\end{tabular}

Table 2. Efficiency of error majorant and minorant for Example 4.3, Case 2.

\begin{tabular}{cccc}
\hline $\boldsymbol{\varepsilon}^{-1}$ & $\boldsymbol{i}_{\oplus}^{\text {eff }}$ & $\boldsymbol{i}_{\ominus}^{\text {eff }}$ & $\boldsymbol{\varkappa}$ \\
\hline 8 & 1.7024 & 0.8291 & 2.0533 \\
16 & 1.9701 & 0.7961 & 2.4750 \\
32 & 2.1848 & 0.7370 & 2.9644 \\
64 & 2.2771 & 0.7124 & 3.1964 \\
\hline
\end{tabular}

Acknowledgment: The authors are grateful to Swiss National Science Foundation for supporting this research under the grants 200021_119809 and 200020_134621. The first co-author also thanks the Institute for Mathematical Research (FIM, ETH, Zurich) for support. We are grateful to Dr. Christian Wüst - the finite element computations have been performed on the basis of his program JCFD.

\section{References}

[1] A. Abdulle, A. Nonnenmacher, A posteriori error analysis of the heterogeneous multiscale method for homogenization problems. C. R. Math. Acad. Sci. Paris, 347 (2009), No. 17-18, 1081-1086.

[2] I. Babuška, W. C. Rheinboldt, A posteriori error estimates for the finite element method, Intern. J. Numer. Math. Engrg., 12 (1978), 1597-1615.

[3] I. Babuška, W. C. Rheinboldt, Error estimates for adaptive finite element computations, SIAM J. Numer. Anal., 15 (1978), 736-754.

[4] I. Babuška, I. Lee, C. Schwab, On the a posteriori estimation of the modeling error for the heat conduction in a plate and its use for adaptive hierarchical modeling, Appl. Numer. Math., 14 (1994), 5-21.

[5] I. Babuška, C. Schwab, A posteriori error estimation for hierarchic models of elliptic boundary value problems on thin domains, SIAM J. Numer. Anal., 33 (1996), 221-246.

[6] N. S. Bakhvalov, G. Panasenko, Homogenisation: Averaging Processes In Periodic Media: Mathematical Problems In The Mechanics Of Composite Materials, Springer, 1989.

[7] A. Bensoussan, J.-L. Lions, G. Papanicolaou, Asymptotic Analysis for Periodic Structures, North-Holland, Amsterdam, 1978.

[8] Z. Cai, S.Zhang, Recovery-based error estimator for interface problems: conforming linear elements, SIAM J. Numer. Anal., 47 (2009), 2132-2156.

[9] M. Chipot, Elliptic Equations: An Introductory Course. Birkhäuser Verlag AG, 2009.

[10] D. Cioranescu, P. Donato, An Introduction To Homogenization, Oxford Lecture Series in Mathematics and its Applications, Vol. 17, Oxford University Press, 1999.

[11] A. Friedman, Partial Differential Equations, R. E. Krieger Pub. Co., Huntington, NY, 1976.

[12] P. Henning, M. Ohlberger, The heterogeneous multiscale finite element method for elliptic homogenization problems in perforated domains, Numer. Math., 113 (2009), No. 4, 601-629.

[13] P. Henning, M. Ohlberger, A-posteriori error estimation for a heterogeneous multiscale method for monotone operators and beyond a periodic setting, Technical Report 01/11-N, FB 10, Universität Münster, 2011.

[14] V. V. Jikov, S. M. Kozlov, O. A. Oleinik, Homogenization of Differential Operators and Integral Functionals, Springer, Berlin, 1994.

[15] R. B. Kellogg, On the Poisson equation with intersecting interfaces, Appl. Anal., 4 (1975), 101-129.

[16] J. T. Oden, J. R. Cho, Adaptive $h p q$-finite element methods of hierarchical models for plate- and shell-like structures, Comput. Meth. Appl. Mech. Engrg., 136 (1996), 317-345.

[17] M. Ohlberger, A posteriori error estimates for the heterogeneous multiscale finite element method for elliptic homogenization problems, Multiscale Model. Simul., 4 (2005), No. 1, 88-114.

[18] L. E. Payne, H. F. Weinberger, An optimal Poincaré inequality for convex domains, Arch. Ration. Mech. Anal., 5 (1960), No.1, 286-292.

[19] S. Repin, A posteriori error estimation for nonlinear variational problems by duality theory, Zapiski Nauch. Semin. (POMI), 243 (1997), 201-214.

[20] S. I. Repin, A posteriori error estimation for variational problems with uniformly convex functionals, Math. Comp., 69 (2000), 481-600.

[21] S. I. Repin, The estimates of the error of some two-dimensional models in the elasticity theory, J. Math. Sci., New York, 106 (2001), 3027-3041. 
[22] S. I. Repin, A posteriori estimates for partial differential equations, Walter de Gruyter, Berlin, 2008.

[23] S. I. Repin and T. S. Samrowski Estimates of dimension reduction errors for stationary reaction-diffusion problems, Problems of Math. Anal., 173 (2011), No. 6, 803-821.

[24] S. I. Repin, T. S. Samrowski, and S. A. Sauter, Combined a posteriori modelling-discretization error estimate for elliptic problems with variable coefficients, ESAIM, Math. Model. Numer. Anal., 46 (2012), No. 6, 1389-1405.

[25] S. I. Repin, T. S. Samrowski, and S. A. Sauter, A posteriori error majorants of the modeling errors for elliptic homogenization problems, C. R., Math., Acad. Sci. Paris, 351 (2013), No. 23/24, 877-882.

[26] S. I. Repin, S. A. Sauter, and A. A. Smolianski, A posteriori error estimation for the Dirichlet problem with account of the error in the approximation of boundary conditions, Computing, 70 (2003), 205-233.

[27] S. I. Repin, S. A. Sauter, and A. A. Smolianski, A posteriori estimation of dimension reduction errors for elliptic problems in thin domains, SIAM J. Numer. Anal., 42 (2004), No. 4, 1435-1451.

[28] T. S. Samrowski, Combined error estimates in the case of the dimension reduction, Preprint 16-2011, Universität Zürich, 2011.

[29] C. Schwab, A-posteriori modeling error estimation for hierarchic plate model, Numer. Math., 74 (1996), 221-259.

[30] Ch. Schwab, A. Matache, Generalized FEM for homogenization problems, in: Multiscale and multiresolution methods, Lect. Notes Comput. Sci. Engrg., 20 (2002), 197-237. Springer, Berlin, 2002.

[31] Ch. Wüst, JCFD: Custom flow simulation in Java, URL: http://www.math.uzh.ch/compmath/jcfd, 2009. 